\title{
A MODEL OF A SIMPLE, BARYON-DOMINATED UNIVERSE THAT EXPANDS AT AN EVER-INCREASING RATE WITHOUT RELYING ON VACUUM ENERGY $(\Lambda)$
}

\author{
GREG P. PROPER \\ Walker, MN 56484, USA \\ gproper@arvig.net
}

Received 7 August 2011

\begin{abstract}
The prevailing view in modern cosmology is that the universe is comprised of immense quantities of exotic materials (i.e. Dark Matter and Dark Energy) that have yet to be positively identified. However, there is also a small group of scientists who believe that the answer to this dilemma is to be found in the modification of gravity (i.e. General Relativity). This short paper states that if we make the bold assumption that all objects/observers are comprised of sets of spacetime coordinates that change (albeit slowly) as the universe ages, then three puzzles that currently confront cosmologists, astronomers and astrophysicists can easily be answered using relatively simple calculations. The condition necessary to explore this possibility can be obtained if one postulates that relativistic gravitational potential lessens (in absolute magnitude) everywhere as the universe ages $(n)$. That is, the spacetime metric $g_{\mu \nu}(\mathbf{x}) \rightarrow g_{\mu \nu}(\mathbf{x}, n)$. If gravity behaves in this manner, then it can be shown that it is the causitive agent of indeterminism in nature.
\end{abstract}

Keywords: Gravitation; cosmology; indeterminism.

PACS Nos.: 04.50Kd, 98.80Jk, 98.80Bp

\section{Introduction}

The fundamental quantities of nature (space, time and mass) that define everything else are invariant within inertial frames (Newtonian physics). However, General Relativity (GR) states that this invariance does not hold with respect to noninertial (i.e. accelerated) frames. The theory espoused in this short paper asserts that if we consider the possibility that GR (i.e. relativistic gravitational potential) changes everywhere as the universe ages, then we can attain significant benefits. This resembles a theory once advanced by P. M. Dirac (i.e. the Large Numbers Hypothesis (LNH)). Dirac's LNH (and its similarity to this theory) is touched upon here (Sec. 2). By way of introduction, this short paper (in the most straightforward manner possible) answers the following question, "What might we observe if we, 
ourselves, reside within a system in which relativistic gravitational potential lessens (in absolute magnitude) everywhere as the universe ages?"

This paper is essentially an introduction to a much longer, more quantitative paper that has been authored with the same title. ${ }^{1}$ For brevity, this paper first introduces this new model of the universe in a qualitative manner. Simple, quantitative analyses that support the conclusions (along with a suggestion for a possible experimental test of the theory) are found in the Appendices. It is hoped that the very simple and limited model developed in this paper will help to introduce the reader to a radically different universe - a very strange universe, yet one that once understood has some very interesting properties.

Specifically, it will be demonstrated that this simplified, cosmological model (with its modified theory of gravity)...

1. Does not require Dark Energy (DE) to expand at a rate that is ever-increasing.

2. Does not require halos of Dark Matter to explain the flattening of galaxy rotational curves (i.e. it corresponds to MOND (MOdified Newtonian Dynamics)). ${ }^{2}$

3. Solves the cosmological constant discrepancy problem (i.e. $\Omega_{\Lambda}$ off by a factor of $\left.10^{120}\right)$.

4. Mandates that a quantifiable indeterminism must underlie nature (Planck's constant $(h)$ can be obtained by direct calculation).

5. Provides an a priori explanation for the equivalence of gravitational mass and inertial mass.

6. Conforms to the conservation laws.

\section{Dirac's Large Numbers Hypothesis}

The author is not the first to conjecture that the universe might, as it ages, change in ways that are not directly perceived and/or understood. Dirac argued that very large dimensionless numbers taking values like $10^{40}$ and $10^{80}$ are most unlikely to be independent and unrelated accidents: there must be some undiscovered mathematical formula linking the quantities involved.

"Any two of the very large dimensionless numbers occurring in Nature are connected by a simple mathematical relation, in which the coefficients are of the order of unity."

P. M. Dirac

The radical change precipitated by Dirac's LNH is that it requires us to believe that a collection of traditional constants of Nature must be changing as the universe ages $(n)$. Dirac chose to accommodate this requirement by abandoning the constancy of Newton's gravitational constant $(G)$. He suggested that it was decreasing in direct proportion to the age of the universe over cosmic time scales, as $G \propto 1 / n$. One now sees that the huge magnitude of the Large Numbers is a consequence of the great age of the universe: they all get larger as time goes on. ${ }^{3}$ 
There are two points that Dirac made with his LNH that are germane to the current discussion: 1 . Certain constants might not be constant after all. They might scale as the universe ages. 2 . If not $G$, then perhaps gravity itself might change as the universe ages. Rather than hypothesize that the gravitational constant $G$ decrease as the universe ages (as Dirac did), the following postulate is advanced for the purposes of this paper. Relativistic gravitational potential $\left(\Phi_{n}\right)$, itself, is decreasing (in absolute magnitude) everywhere as the universe ages $(n)$. The detection of this change in $\Phi_{n}$ may not be as obvious as one would think, because if this change were occurring everywhere, then all change would be relative. In order to explore this possibility further, a new cosmological model (i.e. metric) is needed that provides a mechanism for this type of constant change.

\section{The Model}

The reader is asked to remain mindful of the following facts during the course of reading this short, descriptive paper:

1. Gravity is an acceleration (equivalence principle). Inertial mass is equal to gravitational mass.

2. The atomic model states that atoms are composed of constituent particles that have no discernible volume (i.e. quarks and electrons), and of space (i.e. the vacuum). Objects have substance due to the exclusionary principle. Atoms can be approximated as being spheres with a radius of $\sim 10^{-10} \mathrm{~m}$. In certain aspects, atoms could be considered as vacuous as galaxies.

3. General theories must encompass more restrictive models/theories (correspondence principle).

4. General Relativity has been quantitatively verified only where gravity is relatively strong (i.e. within solar system distances — which are quite small compared to galactic (or greater) distances). ${ }^{2}$

The following is a depiction of expanding spacetime for this model. Expanding spacetime can be visualized as a sequence of infinitesimally thin, nearly concentric shells surrounding the big-bang singularity (Fig. 1). These shells of spacetime become increasingly less dense as one moves outward through them. Spacetime that is less dense can be characterized as spacetime where the space component (i.e. $r$ (the vacuum)) is more expansive (i.e. meter sticks are expanding), and where the time component (i.e. $t$ ) is speeding up (i.e. clocks are running faster). Since temporal dimensions as well as spatial dimensions change in this model universe, the model is relativistic.

A metric for this model can easily be developed by adding a series of constraints to the Robertson-Walker (R-W) metric. The R-W metric for uniformly evolving spacetime is:

$$
d s^{2}=-c^{2} d t^{2}+\left(\frac{R(t)}{R_{0}}\right)^{2}\left(\frac{d r^{2}}{1-k\left(\frac{r}{R_{0}}\right)^{2}}+r^{2}\left(d \theta^{2}+\sin ^{2} \theta d \phi^{2}\right)\right) .
$$




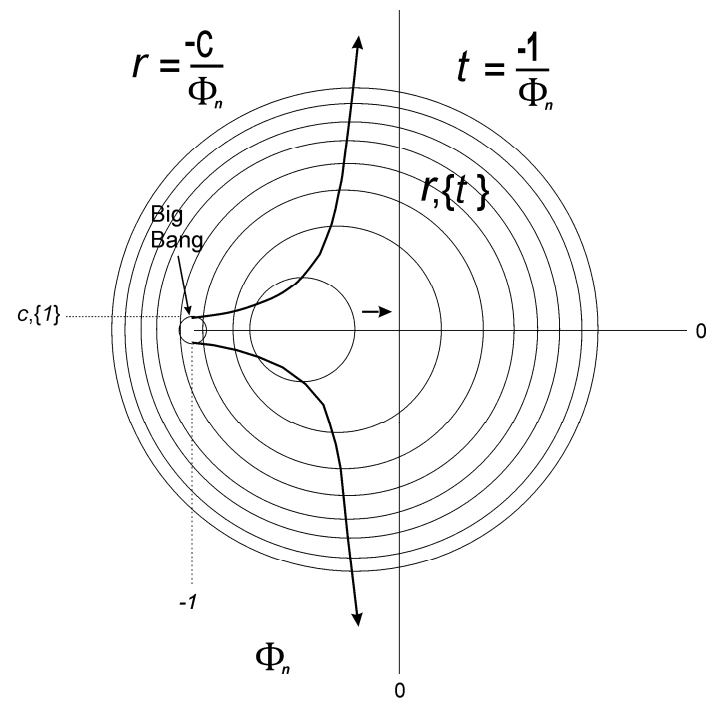

Fig. 1. The Spacetime Shell Model (i.e. hyperbolically expanding Space and Time). [Note: The horizontal scale is exaggerated with respect to the vertical.]

As stated above, in this new model spatial dimensions $(r)$ as well as temporal dimensions $(t)$ both scale as the universe ages $(n)$. That is $r=r(n)$ and $t=t(n)$. Therefore, the scale factor $\left(R(t) / R_{0}\right)$ must be relocated to encompass both the spatial and the temporal components of the R-W metric. [Note: Because of the observer's unique position as a participant in this model, this scaling of time $(t)$ does not result in a violation of Birkhoff's Theorem.] Since practically everything scales in this model (as the universe ages), it is convenient to use Planck units whereby time $(t)$, space $(r)$, gravitational potential $\left(\Phi_{n}\right)$, and various constants of nature all fold into unity (or - unity) at $n=1 t_{P}$. It then becomes possible to scale forward from $n=1 t_{P}$ (rather than backward as with the $\mathrm{R}-\mathrm{W}$ metric). The scale factor function can be simplified by setting $R_{0}=1$ and by setting $R(t)=-1 / \Phi_{n}$. In addition, each spacetime shell in Fig. 1 can be construed as being locally flat (i.e. Minkowskian). Therefore, $k=0$ for each shell (i.e. the curvature of spacetime will be determined entirely by the scale factor). Incorporating these constraints into the R-W metric gives the metric for the Spacetime Shell Model shown in Fig. 1:

$$
d s^{2}=\left(-\frac{1}{\Phi_{n}}\right)^{2}\left(-c^{2} d t^{2}+d r^{2}+r^{2}\left(d \theta^{2}+\sin ^{2} \theta d \phi^{2}\right)\right) .
$$

From Eq. (2) an even more simplified metric can be derived - one that has been reduced to one spatial dimension, and that begins the expansion of spacetime immediately after the big bang event:

$$
d s^{2}=\left(-\frac{1}{\Phi_{n}}\right)^{2}\left(-c^{2} d t_{B B}^{2}+d r_{B B}^{2}\right) .
$$


The physical meaning of the above equation(s) can now be discussed in the context of this model. If the term $\left(-c^{2} d t_{B B}^{2}+d r_{B B}^{2}\right)^{1 / 2}$ can be considered a comoving spacetime distance beginning at $n=1 t_{P}$, then the components of spacetime (i.e. space $(r)$ and time $(t)$ ) can be said to be scaling continually from their initial value of unity at the big bang event (i.e. $t_{B B}=1$ and $r_{B B}=1$ ). In this case, $c$ is just an anthropocentric ratio between expanding space $(r)$ (i.e. meter sticks are expanding everywhere as the universe ages) and increasing time $(t)$ (i.e. clocks are speeding up everywhere as the universe ages). Thus, for this simplified model with one spatial dimension and one temporal dimension (i.e. essentially graphs of $r_{1}$ and $\left.t\right)$ :

$$
\begin{aligned}
r_{1} & =-\frac{c}{\Phi_{n}} \\
t & =-\frac{1}{\Phi_{n}} .
\end{aligned}
$$

Finally, allowing for the fact that nature is discrete at some level, these shells of spacetime are themselves discrete at some infinitesimally small level. The term for relativistic, gravitational potential $\left(\Phi_{n}\right)$ is a sequence. Its allowed positions are:

$$
\Phi_{n}=\left\{-\frac{1}{1},-\frac{1}{2},-\frac{1}{3},-\frac{1}{4}, \ldots,-\frac{1}{n}, \ldots\right\}=\left\{-\frac{1}{n}\right\}_{1}^{\infty} .
$$

[Note: The current age of the universe $(n)$ is $\sim 10^{60} t_{P}$.]

Let us now begin by developing a qualitative description of a very simple model universe which corresponds to the above metric and which adheres to GR. Let us start by invoking the correspondence principle which states that the more general model/theory must incorporate the more restrictive (simplified) model/theory. Therefore, let us start by conceptualizing a simple, Newtonian universe consisting of only two objects. The first object is a point mass (e.g. let us say a certain large mass lying within its Schwarzschild radius - a black hole). The author can apply simple Newtonian physics and state that gravitational potential will vary by $1 / r$ from just outside this point mass/black hole. A second object (let us say a small sphere) can be inserted within this field of gravitational potential, and its physics can be described by either Newtonian gravity or GR. In this very simple universe containing only two objects (where the mass of the centroid object (i.e. the point mass/black hole) is magnitudes greater than the small spherical object) the curvature of spacetime is hyperbolic (i.e. $r$ and $t$ are both proportional to $1 / \Phi_{n}$ (where $\Phi_{n}$ is defined as relativistic gravitational potential) - Fig. 1).

The preceding is a very simplified, gravitational model that incorporates curved spacetime and corresponds to GR. The author will now cause the model to change dynamically as the universe ages - but only in such a manner that it will retain all of the characteristics and benefits of GR (i.e. gravity curving spacetime). In doing so, the model will gain even greater concordance with what is presently known about the universe. The author will now stipulate that the second (much less massive) 
spherical object is actually in a form of motion on a radial path through this field of gravitational potential (and thus through spacetime) in a direction outward from the centroid (i.e. point mass/black hole). [Please note that the author is not describing a movement through space, but rather a specialized movement through spacetime (where the comoving coordinates of the spherical object do not change).] Since this spherical object is moving through lessening (in absolute magnitude) gravitational potentials, this spacetime motion is (in a classical sense) a deceleration (i.e. a negative acceleration). [Note: In this model, differentiating gravitational potential with respect to $r$ (where $r$, itself, (by virtue of the object's radial movement through spacetime) is a function of the age of the universe $(n)$, i.e. $r=r(n))$ gives gravitational acceleration (which in this case is negative).] Thus, in this model, the small, spherical object experiences gravity due to its movement through spacetime.

This decelerative motion (of the spherical object through spacetime) is everdecreasing (in absolute magnitude) based on the field of gravitational potential which, again, varies proportionally to $1 / r$. The author would now point out that GR mandates that spacetime must change as gravitational potential/acceleration changes. (The model universe shown in Fig. 1 has this property.) In order to segue into the next topic, the author will place an observer within the hollow interior of the spherical object (i.e. this spherical object is, in actuality, a type of spacetimeship). The author would now point out that since the object and the observer are moving through this field of lessening (in absolute magnitude) gravitational potential, the spacetime within which the object and the observer reside is also changing (i.e. space is getting more expansive (meter sticks are expanding) and time is speeding up (i.e. clocks are running faster)). The observer is unaware of these changes because he/she is within the system that is changing and is likewise affected (i.e. everything is changing relative to everything else - albeit very slowly). From the observer's perspective the system is static, but, nonetheless, from the perspective of an external, god-like observer space is expanding (meter sticks are lengthening/expanding), time is speeding up (clocks are running faster), and gravitational mass (and thus inertial mass) is diminishing; and all of these things (from the perspective of this godlike observer) are occurring at an ever-increasing (i.e. hyperbolic) rate. Lorentz invariance is maintained as objects move through spacetime.

In the above model, correspondence with GR has been maintained. The author, however, has gained enormous benefits by varying GR (i.e. relativistic gravitational potential) everywhere as the universe ages:

1) Since spacetime naturally exists in a hyperbolic form (Fig. 1), the universe is seen from the perspective of the observer to expand at an ever-increasing rate. It does not require Dark Energy in order to do so (Appendix A).

2) Gravity itself scales everywhere as the universe ages (i.e. not just in the intragalactic void as with MOND). It can be shown that the effects due to this scaling will only be noticeable when gravitational acceleration is very small (i.e. galactic distances and corresponding gravitational accelerations). This corresponds to MOND (MOdified Newtonian Dynamics). ${ }^{2}$ No halos of Dark Matter are necessary to explain the flattening of galaxy rotational curves (Appendix B). 
3) It is common to refer to the energy density of the vacuum as the cosmological constant. The measured value of the cosmological constant $\Omega_{\Lambda}$ is 0.7 , whereas the predicted value is $10^{120}$. This discrepancy has been called, "The worst theoretical prediction in the history of physics." 4 If one considers the possibility that gravity has been scaling (i.e. lessening in absolute magnitude) since the big-bang, then this problem can be solved (Appendix C).

4) In this model, space (the vacuum) and time continually increase as mass continually decreases. This is due to matter continually moving through spacetime that is hyperbolically differential in nature. Another way of stating this is to say that gravitational potential decreases (in absolute magnitude) everywhere as the universe ages. The observer resides within this ever-changing system and thus he/she would not witness this change directly, but he/she would witness this change as an uncertainty in measurements that (as the universe ages (i.e. $\Delta$ )) multiplicatively pair time $(t)$ or space $(r)$ against dynamic quantities that include mass (i.e. energy $(E)$ or momentum $(p)$ ). These dynamic quantities are, themselves, built up from these same fundamental quantities (i.e. space, time and mass). The term velocity $(v)$ in the built-up quantities of energy $\left(1 / 2 m v^{2}\right)$ and momentum $(m v)$ has no bearing on this uncertainty since space (position/length) and time are changing covariantly (i.e. the built-up quantity velocity $(\Delta$ space/ $\Delta$ time) is invariant as space and time both continually increase proportional to $\left.1 / \Phi_{n}\right)$. Thus, in this model, a varying gravitational potential as the universe ages is the cause of an underlying indeterminism in nature (i.e. Uncertainty relationships, themselves, are quantifiable - Planck's constant $(h)$ can be determined by direct calculation - Appendix D). ${ }^{1}$

5) For that same reason (i.e. that velocity and also angular momentum (mvr) are invariant terms in this model (i.e. not functions of $\left.\Phi_{n}\right)$ ), the conservation laws hold and atomic orbits remain stable. Gauss's Law for Gravity states that gravitational flux is proportional to mass only (i.e. the $r^{2}$ terms cancel in Gauss's equation see below), and mass in this model changes (as does space and time) as the universe ages. Thus planetary orbits remain stable also. The integral form of Gauss's Law for Gravity is:

$$
\phi_{g}=\oint g \cdot d S=-\left(\frac{G M}{r^{2}}\right)\left(4 \pi\left(r^{2}\right)\right)=-4 \pi G M
$$

\section{Conclusion}

In the Introduction the author posed the very non-conventional question, "What might we observe if we, ourselves, reside within a system in which the quantities that define our particular reality evolve (in a relativistic manner) as the universe ages?" The analyses in the Appendices show that what we should be observing (on both micro and macro scales) is precisely what we are observing. The author realizes that what the model says about nature (i.e. reality) is that it is very strange indeed - but no stranger than that postulated by some interpretations of Quantum Theory (e.g. many worlds). In the author's companion paper of the same title 
(i.e. A model of a simple, baryon-dominated universe that expands at an everincreasing rate without relying on vacuum energy $(\Lambda)$ ), the author uses this theory to calculate various constants of nature that have, heretofore, only been derived empirically. One example is provided here $(h)$ - Appendix D.

\section{Appendix A. Expansion at an Ever-Increasing Rate without DE}

The model states that gravitational potential lessens (in absolute magnitude) everywhere as the universe ages. Gravitational potential is defined (in this paper) as the Greek character, $\Phi(\operatorname{sub} n)$ :

$$
\Phi_{n}=\text { (relativistic) gravitational potential. }
$$

Based on the model description (Fig. 1) in Sec. 3, both 1D space $\left(r_{1}\right)$ and time $(t)$ can be quantitatively assessed in the following manner (i.e. as functions of gravitational potential $\left.\left(\Phi_{n}\right)\right)$ :

$$
\begin{gathered}
\operatorname{space}\left(r_{1}\right)=-\frac{c}{\Phi_{n}}, \\
\operatorname{time}(t)=-\frac{1}{\Phi_{n}} .
\end{gathered}
$$

[Equations (4) and (5) are analogous to the first part of John Wheeler's famous aphorism, Mass tells spacetime how to curve.]

The graph of spacetime (Fig. 1) defines an expansion of space (and time) that is hyperbolic throughout its domain. Although it is readily apparent by examining Fig. 1 that space $\left(r_{1}\right)$ (and time $(t)$ ) expands (increases) at an ever-increasing rate, a simple mathematical proof is given here:

$$
\begin{array}{r}
r_{1} \propto \frac{1}{\Phi_{n}}, \\
\frac{d r_{1}}{d \Phi_{n}} \propto \frac{1}{\Phi_{n}^{2}} .
\end{array}
$$

As $\Phi_{n} \rightarrow 0$ (Fig. 1), Eq. (A.1) increases at a rate that is ever-increasing.

Thus, the model conforms to recent astronomic observations that have confirmed just such an expansion of space - no vacuum energy is required.

\section{Appendix B. Flattening of Galaxy Rotational Curves without DM}

In this model time $(t)$ is a function of gravitational potential $\left(\Phi_{n}\right)$ :

$$
\text { time }(t) \propto \frac{1}{\Phi_{n}} .
$$

In order to differentiate anthropocentric time (measured in seconds) from cosmic time (measured in Planck (i.e. natural) units $\left(t_{P}\right)$ ), cosmic time will be denoted by $n$. 


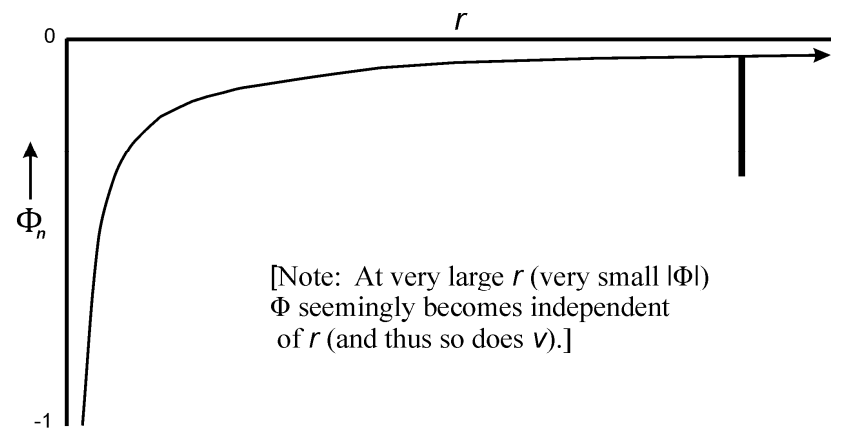

Fig. 2. Graph of $\Phi_{n}$ proportional to $1 / r$. [Note: Fig. 2 is simply Fig. 1 inverted.]

In this model gravitational potential $\left(\Phi_{n}\right)$ changes everywhere as the universe ages. Thus gravitational acceleration $\left(a_{g}\right)$ can be obtained by differentiating gravitational potential $\left(\Phi_{n}\right)$ with respect to cosmic time $(n)$ (i.e. the aging of the universe is the underlying cause of gravity). Equation (5) can be expressed as:

$$
\Phi_{n} \propto \frac{1}{n} .
$$

[In this form Eq. (B.1) is analogous to the second part of John Wheeler's famous aphorism, spacetime tells mass how to move.]

Differentiating Eq. (B.1) gives:

$$
a_{g}(n) \propto \frac{d}{d n} \Phi_{n} \propto \frac{d \Phi_{n}}{d n} \propto \frac{1}{n^{2}} .
$$

Functions such as (B.2) have the unique characteristic of being symmetric about axes. Substituting Eq. (B.1) into Eq. (B.2) gives:

$$
a_{g}(n) \propto \frac{d \Phi_{n}}{d n} \propto \Phi_{n}^{2}
$$

The astronomic observation of galaxy rotational curves has (for large galaxies) shown that there is no Keplerian falloff in velocities. Therefore, galactic rotational velocities seemingly become independent of radius (i.e. at large radii and correspondingly weak gravity). Thus:

$$
a_{g}=\frac{v^{2}}{r} \approx v^{2}
$$

Then (substituting Eq. (B.3) into Eq. (B.4) gives:

$$
\begin{gathered}
v \propto \Phi_{n}, \\
\Delta v \propto \Delta \Phi_{n} .
\end{gathered}
$$

At large $r$ (i.e. weak $a_{g}$ ) $\Delta \Phi_{n} \rightarrow 0$ (Fig. 2). Thus $\Delta v \rightarrow 0$. 
This can be contrasted to the orbits of the planets within the solar system in the following qualitative manner. The heavy dark vertical line that has been added to Fig. 2 would represent the internal gravitational field of the solar system (or any stellar system) that is located far out on the galactic disk (i.e. the galactic gravitational well). Essentially, this divot could be described as a stellar gravitational field (solar system) located within a broader galactic gravitational field. Although the gravitational well of the solar system is also hyperbolic in nature, the $r^{2}$ relationship holds in this instance because $a_{S}$ is quite large with respect to $a_{G}$ (while $r_{S}$ is quite small with respect to $r_{G}$ ). This is illustrated by Fig. 2, and this corresponds to what Milgrom posited when he advanced MOND.

\section{Appendix C. The Solution to the Cosmological Constant Discrepancy}

In this model gravitational acceleration $\left(a_{g}\right)$ can be obtained by differentiating gravitational potential with respect to cosmic time (refer to Appendix B):

$$
a_{g}(n) \propto \frac{d}{d n} \Phi_{n} \propto \frac{d \Phi_{n}}{d n} \propto \frac{1}{n^{2}} .
$$

[Note: $a_{g}$ is a negative acceleration in this model (i.e. $\left.a_{g}=-\nabla \Phi\right)$.] Equation (B.2) can be expressed as:

$$
a_{g}(n)=-\frac{d}{d n} \Phi_{n}=-\frac{d \Phi_{n}}{d n}=-\frac{1}{n^{2}} .
$$

$a_{g}(1)=$ gravitational acceleration at the Big Bang (i.e. at $\left.n=1 t_{P}\right)$ :

$$
a_{g}(1)=-t_{P}^{-2} \text {. }
$$

The age of the universe in Planck units is $\sim 10^{60} t_{P} \cdot a_{g}\left(10^{60}\right)=$ gravitational acceleration at the present age (i.e. at $n=10^{60} t_{P}$ ):

$$
a_{g}\left(10^{60}\right) \approx-10^{-120} t_{P}^{-2}
$$

The ratio of $a_{g}(1) / a_{g}\left(10^{60}\right)=10^{120}$ accounts for the discrepancy in the cosmological constant.

This result can be checked via the equation for the determination of vacuum energy density $\Omega_{\Lambda}$, which is ${ }^{5}$ :

$$
\Omega_{\Lambda} \equiv \frac{\rho_{\Lambda}}{\rho_{c}} \sim\left(\frac{1}{16 \pi^{2}} \frac{E_{P}^{4}}{\hbar^{3} c^{5}}\right)\left(\frac{8 \pi G}{3 H_{0}^{2}}\right) \sim 10^{120} .
$$

Which can be reduced to:

$$
\Omega_{\Lambda} \sim \frac{1}{6 \pi}\left(\frac{c}{l_{P} H_{0}}\right)^{2} \sim 10^{120} .
$$

[Note: In the above equation $l_{P}=$ Planck length.] 
Because of the extreme magnitude of the result, the first term in the above expression is inconsequential. Therefore:

$$
\Omega_{\Lambda} \sim\left(\frac{c}{l_{P} H_{0}}\right)^{2} \sim 10^{120} .
$$

The present value of the Hubble constant (using WMAP values) is the inverse of the age of the universe ${ }^{6}$ :

$$
H_{0}=\frac{1}{t_{0}} .
$$

[Note: This particular model mandates that $H$ (from the perspective of the observer) is constant throughout time (as in an empty universe), and is equal to $1 / t$ at all times. ${ }^{1}$ This is possible because in this model temporal dimensions $(t)$ are also considered to be comoving (i.e. clocks run faster everywhere as the universe ages).]

Substituting Eq. (C.3) into Eq. (C.2):

$$
\Omega_{\Lambda} \sim\left(\frac{c t_{0}}{l_{P}}\right)^{2}=\left(\frac{t_{0}}{t_{P}}\right)^{2} \sim 10^{120} .
$$

[Note: Planck time and a Planck unit of time are numerically equivalent (i.e. $\sim 10^{-43} \mathrm{~s}$ ).] The current age of the universe $n$ (in $t_{P}$ ) is:

$$
n=\left(\frac{t_{0}}{t_{P}}\right) \sim 10^{60} .
$$

Substituting the above into Eq. (C.4) gives:

$$
\Omega_{\Lambda} \sim(n)^{2} \sim\left|a_{g}^{-1}\right| \sim 10^{120}
$$

when $n=10^{60} t_{P}$ - which confirms the first part of the proof.

\section{Appendix D. The Calculation of Planck's Constant (h)}

Planck's constant will be shown to be (in this model) proportional to the slope of the hyperbolic curve at the present estimated age (in anthropocentric units) of the universe over an interval of 1 anthropocentric temporal base unit (i.e. $1 \mathrm{~s}$ ).

In this model universe, the fundamental quantities that define everything else change in a prescribed manner as the universe ages. Therefore, according to this model the fundamental quantity that is time (and the anthropocentric increment by which it is measured - second) must be in a continual state of change, as is the fundamental quantity that is space (i.e. length - meter), and the fundamental quantity that is mass (i.e. kilogram). Indeed, in this model these three fundamental quantities are all changing based on a single parameter $\Phi_{n}$ (i.e. relativistic gravitational potential): 

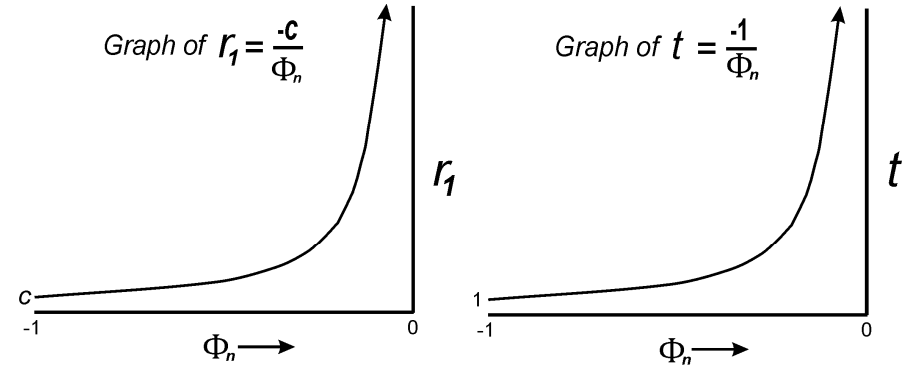

Fig. 3. Graphs of the individual components of spacetime, space $\left(r_{1}\right)$ and time $(t)$.

$$
\begin{gathered}
t=f\left(\Phi_{n}\right)=-\frac{1}{\Phi_{n}}, \\
r_{1}=f\left(\Phi_{n}\right)=-\frac{c}{\Phi_{n}}, \\
m_{0}=f\left(\Phi_{n}\right)=-N m_{B B} \Phi_{n} .
\end{gathered}
$$

Terms for the above equations are defined as follows (definitions will be based on the MKSA system) [Note: By using the generally recognized MKSA system throughout this analysis, the author does not mean to infer that this particular system of measure holds any special prominence in the Universe.]:

Define $\Phi_{n}=$ relativistic gravitational potential $\left(s^{-1}\right)$.

Define $r_{1}=1$-dimensional space (i.e. spatial measurements with 1 degree of freedom) - refer Fig. $3(\mathrm{~m})$.

Define $m_{o}=$ the mass of a random object $(\mathrm{kg})$.

Define $m_{p}=$ the mass of an individual proton (i.e. nucleon) $(\mathrm{kg})$.

Define $N=$ the number of proton equivalents comprising a random object.

Define $m_{B B}=$ an anthropocentric constant resident in all matter (kg-s) - where $m_{p}=f\left(\Phi_{n}\right)=-m_{B B} \Phi_{n}$ and for any object $m_{0}=-N m_{p}$.

Again, since $\Phi_{n}$ varies as the universe ages, then so do $t, r_{1}, m_{p}$ (and $m_{o}$ ) since they are all functions of $\Phi_{n}$.

One might next inquire regarding the disposition of quantities that are built-up from these fundamental quantities — of particular immediate interest is the builtup quantity that is velocity $(v)$. A form of analysis (similar to dimensional analysis) can be employed to determine how these built-up quantities would be affected as this model universe ages. Using this method it can easily be shown that the built-up quantity that is velocity does not change as this model universe ages (i.e. according to this model a quantity (fundamental or built-up) will change as the universe ages only if it is some function of $\left.\Phi_{n}\right)$ :

$$
\operatorname{velocity}(v)=\frac{\Delta r_{1}}{\Delta t}=\frac{m(\text { meter })}{s}=\frac{\Delta\left(-\frac{c}{\Phi_{n}}\right)}{\Delta\left(-\frac{1}{\Phi_{n}}\right)}=c .
$$


Since $r_{1}$ and $t$ are covariant, the built-up quantity velocity $(v)$ is not a function of $\Phi_{n}$ and, therefore, does not change as the universe ages!

The fact that the built-up quantity that is velocity $(v)$ is invariant as this model universe ages (i.e. is not affected as $\Phi_{n}$ decreases in absolute magnitude) would mean that the conservation laws (i.e. mass, energy, linear momentum and angular momentum) would be adhered to in this model universe. [Note: Angular momentum is, like velocity, not a function of $\Phi_{n}$ (which is important for atomic stability).] That is, the fundamental quantity that is mass would be the only quantity changing within the expressions of the conservation laws (i.e. mass, energy, and linear momentum) as the universe ages. Therefore, the relativistic equation for kinetic energy also holds true for this model universe. That is:

$$
E_{K}=E-E_{0}=\left(m-m_{0}\right) c^{2}
$$

where $E_{0}$ (i.e. $m_{0} c^{2}$ ) is equal to an object's initial rest energy.

Energy is conserved as the decrease in a nucleon's rest energy (which occurs as the universe ages) is offset by an increase in its positional energy (i.e. its potential energy becomes less negative). That is:

$$
m_{p} c^{2}+m_{B B} \Phi_{n} c^{2}=0 .
$$

The following equations are Heisenberg's Uncertainty Relationships for timeenergy and position-momentum respectively:

$$
\begin{aligned}
\Delta t \Delta E & \geq h, \\
\Delta r_{x} \Delta p_{x} & \geq h .
\end{aligned}
$$

It is the contention of the author that the $\Delta$ 's in the above expressions, in effect, relate to the changes occurring in the fundamental quantities (i.e. units) as the Universe ages (as described by Eqs. (4), (5) and (D.1)). Heisenberg's time-energy uncertainty relationship can be adapted to this model universe in the following manner:

$$
\begin{gathered}
\Delta t \Delta E \geq h, \\
\Delta t \Delta\left[\frac{1}{2} m v^{2}\right] \geq h .
\end{gathered}
$$

However, in this system (i.e. model universe) from one instant (1 s) to the next, $v$ does not change but $m$ does (i.e. $m$ is a function of $\Phi_{n}$ while $v$ is not).

$$
\Delta t\left[\Delta m\left(\frac{1}{2}\right)\left(v^{2}\right)\right] \geq h .
$$

In this model universe, a unit of mass is changing from one instant to the next based on the parameter $\Phi_{n}$. That is:

$$
\Delta m=-N \Delta m_{p}=-N m_{B B} \Delta \Phi_{n} .
$$


[Note: $m_{B B}$ was previously defined as an intrinsic constant in all matter while $\Phi_{n}$ was previously defined as an intrinsic variable in all matter. $m_{B B}$ is measured in $\mathrm{kg}-\mathrm{s}$ and $\Phi_{n}$ is measured in $\mathrm{s}^{-1}$.]

Equation (D.1) can be substituted into the time-energy uncertainty relationship (i.e. Eq. (D.2)) and the terms that vary from one instant to the next (i.e. $\Delta t$ and $\left.\Delta \Phi_{n}\right)$ can be separated from the intrinsic and external terms that do not vary (i.e. $N, m_{B B}, \frac{1}{2}$, and $v^{2}$ ):

$$
\Delta t\left[\Delta m\left(\frac{1}{2}\right)\left(v^{2}\right)\right]=\Delta t \Delta \Phi_{n}\left((N)\left(-m_{B B}\right)\left(\frac{1}{2}\right)\left(v^{2}\right)\right) \geq h .
$$

Similarly, the Uncertainty equation for position-momentum can be redefined (for this model) as follows (once again, the terms that vary from one instant to the next can be separated from the intrinsic and external terms that do not vary):

$$
\Delta r_{1}[\Delta m(v)]=\Delta r_{1} \Delta \Phi_{n}\left((N)\left(-m_{B B}\right)(v)\right) \geq h .
$$

It should now be further evident to the reader why indeterminism is present in this simple model universe. Quite simply, this model states that a unit of space (i.e. length - a meter), a unit of time (i.e. a second), a unit of mass (i.e. a kilogram) are not what they once were the most minute of instances ago (i.e. they vary as the universe ages). Therefore, if one should pair fundamental quantities (or quantities built-up from these fundamental quantities) that are covariant but changing in a diametric fashion (e.g. time-mass or space (i.e. position)-mass), then, depending on the discreteness of the defined unit used to measure the passage of time (i.e. in this case - 1 second), uncertainty will always be observed. Since observers within this universe are participatory, this uncertainty will only be manifest in multiplicative pairings that include dynamic quantities (i.e. energy $(E)$, momentum $(p)$ ).

The uncertainty in specific measurements is dictated by Eq. (D.3) or Eq. (D.4) and is precisely what the observer should expect to observe if he/she were within a constantly evolving system. This concept can further be illustrated in the following manner. Consider the uncertainty in momentum for each of the two following extreme situations (particle $A$ and object $B$ ) when the uncertainty in position is fixed at the same very miniscule value for both situations (e.g. say $\Delta x=10$ Fermi - the approximate size of a large nucleus). Given that:

$$
m_{A} \ll m_{B} \text { and } \quad p_{A} \ll p_{B} .
$$

If the uncertainty in position for both situations is fixed at the same miniscule value $(\Delta x)$, then

$$
\Delta p_{A} \geq\left(\frac{h}{\Delta x}\right) \quad \text { and } \quad \Delta p_{B} \geq\left(\frac{h}{\Delta x}\right)
$$

In which case the limit of the precision in the measurement of the momentum would be:

$$
\Delta p_{A}=\Delta p_{B}=\Delta p
$$


This would then mean that:

$$
\left(\frac{\Delta p}{p_{B}}\right) \ll\left(\frac{\Delta p}{p_{A}}\right) \text { and }\left(\frac{\Delta p}{m_{B}}\right) \ll\left(\frac{\Delta p}{m_{A}}\right) .
$$

Under this scenario it could be entirely possible that the \% uncertainty in momentum for particle $A$ could be significant; whereas, it could be equally possible that $\Delta p$ could be literally swamped by the immensity of object $B$ 's momentum which, in turn, would mean that the $\%$ uncertainty in object $B$ 's momentum could be indiscernible (which is the normal case for macro-sized objects). However, the limiting factor by which the precision in the magnitude of the momentum is knowable is the same for both situations (i.e. $h$ ). This would indicate that the terms in parentheses in Eq. (D.4) (i.e. $(N)\left(-m_{B B}\right)(v)$ ) have no bearing on the intrinsic change within an object (i.e. a system of infinitesimally small particles separated by vacuum) where this change is manifest in the uncertainty measurement of dynamic quantities. The only term that is germane to the uncertainty calculations would be the constantly diminishing (in absolute magnitude) gravitational potential (i.e. $\Phi_{n}$ ) that (according to this model) is built into all matter. [Note: This line of reasoning (i.e. Eq. (D.1)) also provides an a priori explanation for the equivalence of gravitational and inertial mass.]

The reader should again consider the following uncertainty relationships for this model:

$$
\begin{gathered}
\Delta t \Delta \Phi_{n}\left((N)\left(-m_{B B}\right)\left(\frac{1}{2}\right)\left(v^{2}\right)\right) \geq h, \\
\Delta r_{1} \Delta \Phi_{n}\left((N)\left(-m_{B B}\right)(v)\right) \geq h .
\end{gathered}
$$

These uncertainty relationships measure an intrinsic change that is occurring in the fundamental quantities of space, time, and mass as the universe ages. Again, the terms in parenthesis are intrinsic or external constants which can have no bearing on the limit of certainty which is $h$. The author would also point out that the above equations are dimensionally correct based on the definitions of $\Phi_{n}$ and $m_{B B}$.

Because the limiting factor in the uncertainty equations (i.e. Planck's constant $h$ ) is not affected by the size or momentum of the object in question, it would appear that the terms that change can be isolated from the intrinsic (and external) terms that are constants - in which case a mathematical solution for $h$ can be obtained. [Note: it must be remembered, however, that these constants remain present in these relationships, and that these terms carry the dimensions necessary for dimensional accuracy.]

If the terms that do not vary as the universe ages are excluded, then Eqs. (D.3) and (D.4) can be reduced to the following forms:

$$
\begin{aligned}
& \Delta t \Delta \Phi_{n} \geq h, \\
& \Delta r_{1} \Delta \Phi_{n} \geq h .
\end{aligned}
$$




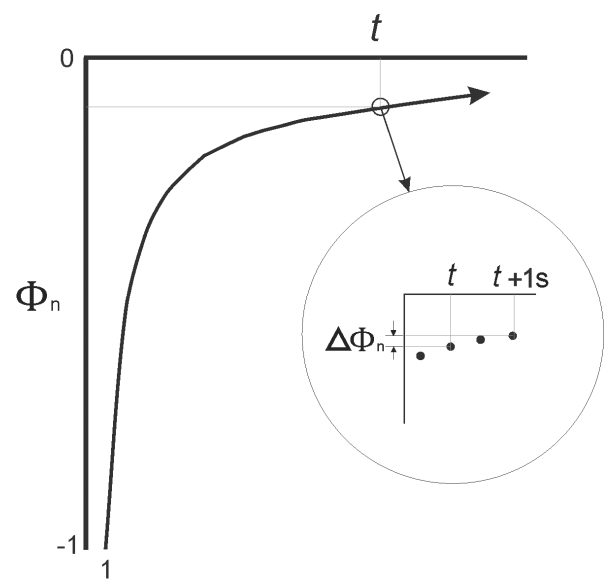

Fig. 4. The uncertainty relationship.

The above equations (i.e. Eqs. (D.3a) and (D.4a)) demonstrate that a causal uncertainty (indeterminism) underlies this model universe. A solution to these equations can be easily derived by formulating a simple differential. The reader should consider the following inverse relationship (Fig. 4):

$$
\begin{array}{r}
\Phi_{n}=-\frac{1}{t}, \\
\Delta \Phi_{n}=\frac{1}{t^{2}} \Delta t .
\end{array}
$$

Equation (D.5) indicates that $\Delta \Phi_{n}$ must change as the universe ages (inversely, anthropocentric time is a function of $\Phi_{n}$ ). Equation (D.5) can be substituted into Eq. (D.3a):

$$
\Delta t \Delta \Phi_{n} \geq h \rightarrow \Delta t\left[\frac{1}{t^{2}} \Delta t\right] \rightarrow\left[\frac{\Delta t^{2}}{t^{2}}\right] \geq h .
$$

The limiting value of this general expression is:

$$
h=K\left[\frac{\Delta t^{2}}{t^{2}}\right] \text {. }
$$

[Note: The above expression is dimensionless ( $K$ is a dimensionless constant). The units necessary for dimensional accuracy lie within the invariant terms.]

The above equation (Eq. (D.6) basically states that there is a general relationship between Planck's constant $(h)$ and the age (in anthropocentric units) of the one-dimensional, relativistic universe developed in this paper.

Since $r_{1}=c t$ (Eqs. (4) and (5), this general expression for $h$ can also be expressed as:

$$
h=K\left[\frac{\Delta r_{1}^{2}}{r_{1}^{2}}\right]
$$




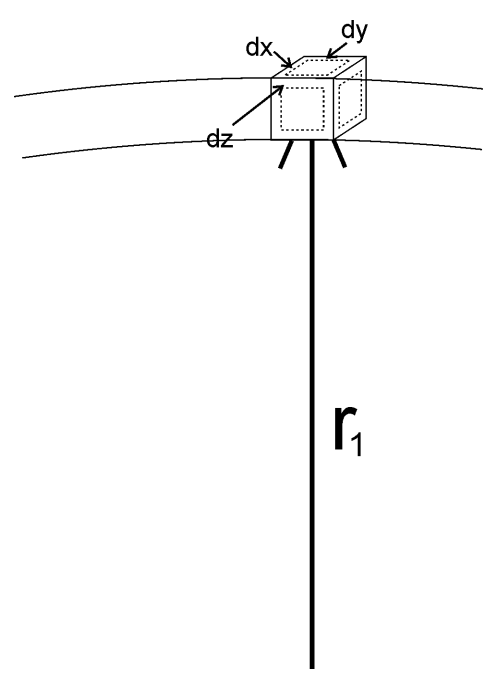

Fig. 5. A unit block of expanding vacuum.

The reader should pause for a moment to further consider Fig. 4 along with the information presented thus far. The fundamental quantities that are spatial (i.e. length) and temporal (i.e. time), and that define matter (i.e. mass) are changing continually within this model universe in accordance with the relativistic relationships that were established earlier. If one would try to simultaneously measure certain pairs of variables (e.g. position and momentum), one would always find an uncertainty, because in the briefest instant, a meter, a second, and a kilogram are not what they once were the minutest of instances ago. Please do not be confused here. The author is not talking about a change in the quantity of an item. Rather, the author is stating that measures are changing, clocks are changing, and all devices and instruments that are used to make a record are themselves changing (unbeknownst to us) because the underlying fabric (i.e. spacetime) that defines reality is constantly changing.

Equation (D.7) allows for a movement in one dimension only (i.e. 1 degree of freedom - Fig. 3). However, experiments where $h$ is empirically derived take place in 3D space. The shell model (Fig. 1) can be used to help derive the final form of the equation used in the determination of $h$ (Fig. 5 is a variant of Fig. 1). In this instance a unit block of expanding vacuum within a single, spacetime shell is depicted. The reader will note that space within the shell expands in three dimensions (i.e. three degrees of freedom). This unit block of $3 \mathrm{D}$ vacuum can be described as having its own local coordinates which scale solely on the length of $r_{1}$ (a scalar variable).

Based on this description, Eq. (D.7) can be modified for three spatial degrees of freedom to:

$$
h=\left[\frac{\Delta l^{2}}{r_{1}^{2}}\right] .
$$


From Fig. 5:

$$
d l^{2}=d x^{2}+d y^{2}+d z^{2} .
$$

In a universe that is isotropic and homogeneous, the expansion of spacetime must be symmetrical about any point. Therefore:

$$
d x \approx d y \approx d z
$$

It is also evident from Fig. 5 that:

$$
d x=2 \pi d r_{1} .
$$

Therefore,

$$
\begin{gathered}
d l^{2}=(3)\left(2 \pi d r_{1}\right)^{2}=12 \pi^{2} d r_{1}^{2}, \\
\left\{d l=2 \sqrt{3} \pi d r_{1}\right\}, \\
K=12 \pi^{2} .
\end{gathered}
$$

Equations (D.6) and (D.7) can now be expressed as:

$$
\begin{aligned}
& h=12 \pi^{2}\left[\frac{\Delta t^{2}}{t^{2}}\right], \\
& h=12 \pi^{2}\left[\frac{\Delta r_{1}^{2}}{r_{1}^{2}}\right] .
\end{aligned}
$$

[Note: If the vacuum expands everywhere as has been hypothesized by this author and incorporated into this model (and since atoms consist almost entirely of vacuum), then all objects expand as the universe ages (while time continually speeds up).]

Lummer and Pringsheim's recording of the emissive power from a black body, and Millikan's experiment confirming Einstein's Theory of the Photoelectric Effect were some of the first experiments in which $h$ was measured. These experiments occurred in three-dimensional space, what was measured was either $\lambda$ (momentum) or $\nu$ (energy), and the system of units that was used in these experiments was the MKSA system (i.e. the second). The same anthropocentric base unit (i.e. the second) that was used by the experimentalists in their work must be used in calculating $h$. Therefore, $\Delta t$ in Eq. (D.6) is the unit that is used to measure time elapsing in experiments where $h$ is empirically derived (i.e. $\Delta t=1 \mathrm{~s}$ ).

The accepted age of the Universe is $13.7 \times 10^{9}$ years. The age of the Universe $(t)$ in seconds is:

$$
\left(13.7 \times 10^{9} \mathrm{yr}\right)(365 \text { day } / \mathrm{yr})(24 \mathrm{hr} / \text { day })(3600 \mathrm{~s} / \mathrm{hr})=4.32 \times 10^{17} \mathrm{~s} .
$$


Then:

$$
h=12 \pi^{2}\left[\frac{(1 \mathrm{~s})^{2}}{\left(4.32 \times 10^{17} \mathrm{~s}\right)^{2}}\right]=6.34 \times 10^{-34} \mathrm{~kg}-\mathrm{m}^{2} / \mathrm{s} .
$$

Again, although the uncertainty relationship(s) have been simplified to consider only those variables that change from one position in spacetime to the next, the units for the invariant terms (i.e. $m_{B B}$ and $v^{2}$ ) must be carried along for dimensional accuracy. This occurs because of the unique position of the observer who is, in effect, conducting experiments within a system that is constantly changing. Essentially, these units of measure are baked into the various experiments in which Planck's constant is empirically obtained (i.e. Ammeters $(C / s)$ and voltmeters $(N-m / C)$ are used in making records).

This above result is within $4 \%$ error of the experimentally measured value of Planck's constant $(h)$ (which is negligible when considering the magnitude of the exponent (i.e. $\left.10^{-34}\right)$ ). The error stated is also reasonable considering that the derivation is based on the age of the Universe. The reader must also consider that our world is not at the leading edge of spacetime. Consequently, although the Universe is $\sim 13.7$ eons old, our world is not at that most advanced position (shell) in spacetime (i.e. some matter leads and some matter trails in its passage through spacetime).

Dirac's Constant $(\hbar)$ follows logically from the above derivation of Planck's Constant $(h)$. In order to understand this logical progression, consider for a moment the Bohr atom. The equation $n \hbar=m v r$ is Bohr's angular momentum postulate (i.e. orbital angular momentum is quantized based on multiples of $\hbar$.). It just so happens that angular momentum is (within the context of this model) a rather unique, built-up, physical quantity (similar to velocity) in that it is invariant across different positions in spacetime (i.e. it is not a function of $\Phi_{n}$ ). It can easily be proven that the built-up quantity that is angular momentum is invariant across spacetime positions by performing an analysis similar to that performed for velocity.

$$
|m v r| \rightarrow\left|\left(-m_{B B} \Phi_{n}\right) \frac{\left(-\frac{c}{\Phi_{n}}\right)}{\left(-\frac{1}{\Phi_{n}}\right)}\left(-\frac{c}{\Phi_{n}}\right)\right|=m_{B B} c^{2},
$$

where $m_{B B}$ and $c$ are constants.

Since all five of the quantum numbers (i.e. those that define the radius, the eccentricity, and the orientation of an electron in orbit around a nucleus, as well as those that define the spin, and the spin orientation of the electron itself) are all dependent on either orbital or spin angular momentum, the fact that angular momentum is an invariant quantity (i.e. it does not change due to the movement of matter through differential spacetime) explains the stability of atoms (as well as the quantization of various constants of the motion within atoms). Angular momentum is defined by radial measurements and not by measurements made on the surface of a sphere. Therefore, within the context of the model (i.e. Fig. 1): 


$$
\frac{\hbar}{\text { radial measure }}=\frac{h}{\text { spherical surface measure }} \text {. }
$$

That is:

$$
\frac{\hbar}{r_{3}}=\frac{h}{l} \rightarrow \hbar=\frac{r_{3}}{l} h
$$

However, $l=2 \pi\left(r_{3}\right)$. Substituting gives

$$
\hbar=\frac{r_{3}}{2 \pi\left(r_{3}\right)} h=\frac{h}{2 \pi} .
$$

The model (metric) provides a physical relationship between Planck's constant $h$ and Dirac's constant $\hbar$.

\section{Appendix E. A Possible Means to Experimentally Test the Theory}

Equation (D.1) states that a relativistic gravitational potential $\Phi_{n}$ that lessens (in absolute magnitude) as the universe ages is built into all matter. That is:

$$
\begin{gathered}
m_{0}=f\left(\Phi_{n}\right)=-N m_{B B} \Phi_{n}, \\
m_{p}=-m_{B B} \Phi_{n}, \\
m_{p} c^{2}+m_{B B} \Phi_{n} c^{2}=0 .
\end{gathered}
$$

The allowed states of $\Phi_{n}$ are:

$$
\Phi_{n}=\left\{-\frac{1}{1},-\frac{1}{2},-\frac{1}{3},-\frac{1}{4}, \ldots,-\frac{1}{n}, \ldots\right\}=\left\{-\frac{1}{n}\right\}_{n=1}^{\infty} .
$$

As stated previously, Eq. (D.1) (in this form) also provides an a priori reason for the equivalence of inertial and gravitational mass.

The theory also states (based on the equivalence principle) that an acceleration actually displaces an object in spacetime. Therefore, if the means could be devised to accelerate (displace) an object (i.e. particle) to a high velocity, retain that high rate of velocity for a period, followed by a deceleration and return of the particle to its original state (while carefully measuring the inputs), one would find a very small differential in the energies required for the acceleration as opposed to the deceleration (i.e. the deceleration would consume slightly more energy).

\section{References}

1. G. P. Proper, A model of a simple, baryon-dominated universe that expands at an everincreasing rate without relying on vacuum energy $(\Lambda)$, http://www.nodarkenergy.com/ nodarkenergy.pdf.

2. M. Milgrom, Astrophys. J. 270, 365 (1983). 
3. J. D. Barrow, The Constants of Nature (Pantheon Books, 2002), pp. 99-101.

4. M. P. Hobson, G. P. Efstathiou and A. N. Lasenby, General Relativity: An Introduction for Physicists (Cambridge Univ. Press, 2007), p. 187.

5. T.-P. Cheng, Relativity, Gravitation and Cosmology, 2nd edn. (Oxford Univ. Press, 2010), p. 272

6. B. W. Carroll and D. A. Ostlie, An Introduction to Modern Astrophysics, 2nd edn. (Addison-Wesley, 2007), p. 1058. 